\title{
Multi-objectivization of Short-Term Unit Commitment under Uncertainty using Evolutionary Algorithm
}

\author{
Anupam Trivedi, Deepak Sharma and Dipti Srinivasan \\ Department of Electrical and Computer Engineering \\ National University of Singapore (NUS), Singapore-117576 \\ E-mail: eleat@nus.edu.sg, eledeepa@nus.edu.sg, elesd@nus.edu.sg
}

\begin{abstract}
The short-term unit commitment problem is traditionally solved as a single-objective optimization problem with system operation cost as the only objective. This paper presents multi-objectivization of the short-term unit commitment problem in uncertain environment by considering reliability as an additional objective along with the economic objective. The uncertainties occurring due to unit outage and load forecast error are incorporated using loss of load probability (LOLP) and expected unserved energy (EUE) reliability indices. The multiobjectivized unit commitment problem in uncertain environment is solved using our earlier proposed multi-objective evolutionary algorithm [1]. Simulations are performed on a test system of 26 thermal generating units and the results obtained are benchmarked against the study [2] where the unit commitment problem was solved as a reliability-constrained single-objective optimization problem. The simulation results demonstrate that the proposed multi-objectivized approach can find solutions with considerably lower cost than those obtained in the benchmark. Further, the efficiency and consistency of the proposed algorithm for multi-objectivized unit commitment problem is demonstrated by quantitative performance assessment using hypervolume indicator.
\end{abstract}

Keywords-Multi-objectivization, Unit commitment, Evolutionary algorithms, Multi-objective optimization

\section{NOMENCLATURE}

$T_{\max } \quad$ number of hours in dispatch period

$t \quad$ hourly time index

$N \quad$ number of generating units

$i \quad$ generating unit index

$P_{i}^{t} \quad$ power generated by unit $i$ at hour $t$

$P_{\max , i} \quad$ rated upper limit generation of unit $i$

$P_{\text {min }, i} \quad$ rated lower limit generation of unit $i$

$f_{i}^{t} \quad$ fuel cost of $i$ th unit at hour $t$ in $\$ / \mathrm{h}$

$S U_{i}^{t} \quad$ start-up cost of unit $i$ at hour $t$

$S D_{i}^{t} \quad$ shut-down cost of unit $i$ at hour $t$

$L^{t} \quad$ load demand at hour $t$

MUT/MDT minimum up/down time of unit $i$

$a_{i}, b_{i}, c_{i} \quad$ fuel cost coefficients of the $i$ th generator

$a_{l i}, b_{l i}, c_{l i} \quad$ emission cost coefficients of the $i$ th generator

\section{INTRODUCTION}

The Unit Commitment (UC) problem plays an important role in the daily operation planning of power systems. It involves two scheduling decisions, namely, the "unit commitment" decision and the "electrical power dispatch" (or load dispatch) decision. The "unit commitment" decision involves determining the on/off state of the generators while the "electrical power dispatch" decision involves distributing the forecasted load demand among the committed generators for each hour of the planning horizon. The UC problem requires effectively performing the above two decisions to meet the forecasted load demand over the planning horizon, satisfying a large set of operating equality and inequality constraints and achieving certain objectives. Traditionally, the UC problem has been solved in the literature considering system operation cost as the only (economic) objective while few studies have considered the important criteria, reliability as constraint. Moreover, the UC problem involves uncertainty in generator availability and load forecast data. However, the UC problem is usually solved in deterministic environment. System operators would nevertheless prefer to obtain more reliable generation schedule by incorporating various uncertainties.

The UC problem is a non-linear, large scale, mixed-integer combinatorial, constrained optimization problem. The exact solution to the problem can be obtained only by complete enumeration at the cost of large computational time requirement for realistic power systems. Therefore, over the last two decades, considerable research has been focused on obtaining efficient, near-optimal solutions using AI techniques. The techniques include genetic algorithm (GA) [34], memetic algorithm (MA) [5], particle swarm optimization (PSO) [6-7] and simulated annealing (SA) [8].

As mentioned earlier, the UC problem is generally solved as a single-objective optimization problem. However, better solution to UC problem can be obtained by adopting the concept of multi-objectivization. According to the literature, multi-objectivization is the method of reformulating a SOOP as a MOOP by decomposition of the original objective into sub-objectives or by addition of new objectives and solving it with a multi-objective optimization algorithm, in order to obtain a solution to the original SOOP [9]. The re-formulated 
problem is then most commonly solved using multi-objective evolutionary algorithms (MOEAs).

The study by Knowles et al. [10] that introduced the concept of multi-objectivization of SOOP via decomposition of the original objective indicated that multi-objectivization can reduce local optima and facilitate improved optimization in some cases. The work by Jensen [11] demonstrated that the use of helper objectives (additional objectives guiding the search) significantly improves the standard performance of a standard GA on SOOP. Recent research has reinforced the concept of multi-objectivization proposed by both Knowles et al. [10] and Jensen [11] that multi-objectivization of a SOOP can enhance the search performance and provide improved results. The algorithms based on both the concept of decomposition of objective as well as the use of helper objectives have demonstrated better performance than singleobjective methods for N-P hard problems like the traveling salesman problem (TSP) [11],[12] and the job shop scheduling problem (JSSP) [11].

Handl and Knowles [13] found that better solutions are obtained using a bi-objective formulation of clustering problem by simultaneously minimizing the intra-cluster distance and maximizing the inter-cluster distance than using the usual single-objective minimization of the ratio of the intra-cluster distance to the inter-cluster distance. Coello Coello [14] proposed the approach to solve constrained SOOP by converting the task into a two-objective optimization task of additionally minimizing an aggregate constraint violation and found the approach to work better on several test problems than SOO method. Watanabe and Sakakibara [15] proposed two promising approaches for multi-objectivization by addition of new objectives based on relaxation of the constraints of the problem and addition of noise to the original objective value or decision.

In this paper, the short-term UC problem in uncertain environment is multi-objectivized by using reliability as a helper-objective. The multi-objectivized UC problem is solved using our earlier proposed multi-objective evolutionary algorithm [1]. The main contribution of this paper is in demonstrating the advantage of multi-objectivizing the UC problem by adding reliability as a helper-objective. The proposed multi-objectivized approach is tested on a system of 26 thermal generating units and the results obtained are benchmarked against the study [2] where the UC problem was solved as a reliability-constrained single-objective optimization problem.

The multi-objectivized UC problem is formulated in Section III. Thereafter, the proposed algorithm is described in Section IV. The results and discussions are presented in Section V and the paper is concluded in Section VI.

\section{Multi-OBJeCtivizATION OF Unit COMMITMENT: PROBLEM FORMULATION}

In this section, the primary and the helper objective in the re-formulated UC problem and the associated constraints are presented.

\section{A. Objective Functions}

1) Primary Objective - System Operation Cost:

The total system operating cost includes the fuel cost of the committed generators and the transition cost over the entire scheduling horizon.

$F_{1}=\sum_{t=1}^{T_{\max }} \sum_{i=1}^{N} f_{i}^{t}+S U_{i}^{t}+S D_{i}^{t}$

The fuel cost $f_{i}^{t}$ of unit $i$ is expressed as the quadratic function of its power output during hour $t$.

$f_{i}^{t}=a_{i}\left(P_{i}^{t}\right)^{2}+b_{i}\left(P_{i}^{t}\right)+c_{i}$

The transition cost is the sum of start-up costs and shut-down costs.

\section{2) Helper Objective - Reliability}

In this paper, the expected unserved energy (EUE) index which expresses the expected amount of energy not supplied by the generation system during the scheduling horizon is used as a measure of reliability of the system. The objective function $F_{2}$ is equal to the EUE index itself. It is noted that the reliability of the system is inversely proportional to the EUE index value i.e., the lower the EUE index value, the higher is the reliability of the system. The procedure for calculating the EUE index is presented later in the paper.

The reason for choosing EUE index as a helper objective is that the EUE index is conflicting in nature with system operation cost. This conflicting nature can be explained by a small example. A system can be made more and more reliable (with a lower EUE index value) by committing more and more generating units i.e., by increasing the system operation cost. In multi-objectivization, when the helper-objective conflicts with the primary objective, the helper-objective helps maintain diversity in the population and guides the search away from local optima [11].

\section{B. Constraints}

The solution must satisfy several system and unit related constraints as follows:

1) System power balance: the total power generation at hour $t$ must be equal to the load demand for that hour.

Hence,

$\sum_{i=1}^{N} P_{i}^{t}=L^{t}, \quad t=1,2, \ldots, T_{\max }$

2) Unit minimum up/down time: if a unit is off, it must remain off for at least MDT time duration. In the same way, if a unit is on it must remain on for at least MUT time duration.

3) Unit generation limits: for stable operation, the power output of each generator is restricted within lower and upper limits as follows:

$P_{\min , i} \leq P_{i}^{t} \leq P_{\max , i}, \quad i=1,2, \ldots, N$

4) Reliability constraint - The LOLP reliability constraint is incorporated as:

$L O L P_{t} \leq L m a x, \quad t \in[1, T]$ 
where $L O L P_{t}$ is the LOLP for hour $t$ and Lmax is the user defined maximum allowed limit of the LOLP reliability index.

\section{Proposed MoEA For Multi-ObJectivized Unit COMMITMENT PROBLEM UNDER UNCERTAINTY}

In this paper, our earlier proposed multi-objective evolutionary algorithm [1] has been implemented to solve the multi-objectivized UC problem in uncertain environment. It is noted that our earlier proposed algorithm is referred as MOUCA (Multi-objective unit commitment algorithm) in this paper. The uncertainties due to unit outage and load-forecast error have been incorporated in the solution with the help of reliability indices LOLP and EUE which are defined as follows.

- The LOLP index expresses the probability that the generation system will not cover the forecasted demand.

- The EUE index expresses the expected amount of energy not supplied by the generation system during the scheduling horizon.

The details of MOUCA and the calculation of LOLP and EUE indices are presented in the following sections.

\section{A. Chromosome Formulation}

The UC problem deals with both discrete (binary) and continuous (real) variables. The binary variables represent the operating status (on/off) of the units and the continuous real variables represent the hourly thermal power dispatch of the units. For every chromosome, a $N \times T_{\max }$ binary unit commitment matrix (UCM) is used to represent the generator on/off status and a $N \times T_{\max }$ real power matrix (RPM) is used to represent the corresponding power dispatch. It is noted that a chromosome's actual generation schedule is represented by its resultant power matrix (Res.PM) which is obtained by multiplying the corresponding elements of UCM and RPM of the chromosome.

\section{B. Availability of Generating Units}

Each generating unit is considered as a two-state model, according to which a unit is either available or unavailable for generation. According to this model, the unavailability of the generating unit $i$ during a short time interval $L T$ (known as the system lead time) is given by

$$
U_{i}(L T)=1-e^{-\lambda_{L} L T}
$$

where $\lambda_{i}$ is the failure rate of unit $i$ [2]. The probability $U_{i}(L T)$ given by (6) is known as the outage replacement rate (ORR) of the unit.

\section{Calculation of LOLP and EUE Indices}

To calculate the LOLP and EUE indices for every chromosome, the conventional "loss of load" method is used. This method is based on the convolution of the capacity outage probability table (COPT) with the given load curve [2]. The LOLP index for each hour $t$ is calculated as
$L O L P_{t}=\sum_{j=1}^{n} P R_{j} L_{O S S}, \quad t \in[1, T]$

where $L O S S_{j}$ is given by

LOSS $_{j}=\left\{\begin{array}{l}1, \text { if } C R_{j}<L O A D_{t} \\ 0, \text { otherwise }\end{array}\right.$

Similarly, the EUE index for each hour $\mathrm{t}$ is calculated as

$$
E U E_{t}=\sum_{j=1}^{n} P R_{j} \operatorname{LOSS}_{j}\left(L O A D_{t}-C R_{j}\right), \quad t \in[1, T]
$$

The EUE index of the dispatch period is given by $E U E_{\text {tot }}$ which is calculated as

$E U E_{t o t}=\sum_{t=1}^{T} E U E_{t}$

The computational time in creating capacity outage probability table (COPT) is reduced by omitting the outage levels for which the cumulative probabilities are less than a predefined limit, e.g., $10^{-7}[2]$. Additionally, to avoid the need for repeated creation of COPT, a memory archive is created to store the commitment patterns for each time period, and their corresponding LOLP and EUE index values. In subsequent generations, whenever a commitment pattern is encountered for which there is already an entry in the memory archive, the corresponding LOLP and EUE values are copied and assigned to the repeated pattern. This technique significantly reduces the computational time. It is noticed that a bottle neck still exists in evaluating the reliability indices of a chromosome. Hence, for further improvement in the computational speed of MOUCA, parallel computing is implemented (using openMP) and the hourly LOLP and EUE values of a chromosome are computed in parallel.

\section{Inclusion of Load Forecast Uncertainty}

Load forecast may be associated with a large uncertainty and hence plays a major role in the solution of the UC problem. In this paper, the load forecast uncertainty is assumed to be normally distributed [2] and is represented by a seven-step approximation $(0, \pm 1 \sigma, \pm 2 \sigma, \pm 3 \sigma)$ to the normal distribution. The standard deviation ( $\sigma$ or $S$ ) is a percentage of the expected demand (distribution mean) and is suggested to be $5 \%$ [16]. With the assumption of load forecast uncertainty to be normally distributed, the LOLP and EUE index calculation for each hour $t$ is given by the following equations:

$$
\begin{aligned}
& L O L P_{t}=\sum_{m=1}^{7} \operatorname{LOLP}_{t}(m) P L(m), \quad t \in[1, T] \\
& E U E_{t}=\sum_{m=1}^{7} E U E_{t}(m) P L(m), \quad t \in[1, T]
\end{aligned}
$$

Where $P L(m)$ indicates the probability associated with the discrete interval $m$ in the seven-step model. Similarly, $\operatorname{LOLP}_{t}(m)$ and $E U E_{t}(m)$ represent the LOLP and EUE for each discrete interval $m$ for hour $t$.

\section{E. Generation of Initial Population}

All chromosomes in the initial population are generated 
randomly. UCM of each chromosome is a randomly generated binary matrix. In RPM of each chromosome, the power dispatch of each generator is generated randomly within the power limits of that generator.

\section{F. Constraint Handling and Function Evaluation}

For each chromosome, a COPT is created for every hour $t$ followed by calculation of LOLP and EUE index values. The LOLP and EUE constraint violation $(\mathrm{CV})$ are then calculated using the following equations where $P_{1}$ and $P_{2}$ represent the penalty factors.

$$
L O L P C V=\sum_{\text {time }=1}^{\text {Tmax }} P_{1}(L O L P[\text { time }]-L m a x)
$$

To evaluate the violation of other constraints, the corresponding elements of UCM and RPM of each chromosome are multiplied to form resultant power matrix (Res.PM) which represents a chromosome's actual generation schedule. The minimum up-down time and load demand equality constraint violation are calculated for each chromosome using its Res.PM.

If a chromosome violates the load demand equality constraint, then it is repaired using a heuristics based repair operator. The chromosomes are repaired using priority list (PL) [17] based on fuel cost coefficients. The details of the repair operator can be found in our earlier study [1]. The load demand constraint violation is taken as the absolute value of constraint violation whereas for calculating the minimum updown time constraint violation, a penalty value of $P_{3}$ is imposed for every time the constraint is violated. The constraint violation of minimum up-down time, load demand and LOLP are then summed up to calculate the total amount of constraint violation of a chromosome. A chromosome is feasible if the total constraint violation is less than $10^{-5}$. After constraint violation evaluation, the objective function system operation cost $\left(F_{l}\right)$ is calculated for each chromosome using its Res.PM while objective function $F_{2}$ is equal to the EUE index $\left(E U E_{t o t}\right)$.

\section{G. Ranking and Selection}

The chromosomes are ranked using the constraineddomination principle. Thereafter, constrained-binary tournament method is used as the selection method and is based on the same principles as the constrained-domination condition [18].

\section{H. Crossover}

The UC problem involves both discrete binary variables and continuous real variables. Hence, binary and real crossover operators are employed to explore both the search spaces. Standard crossover operators do not work well on the binary variables in the generation scheduling problem [5]. In this paper, a slightly modified version of the window crossover operator as mentioned in [5] is used as the binary crossover. It works by randomly selecting two parents from the mating pool and then randomly selecting a window size. The unit commitment entries within the window portion are exchanged between the UCM of two parents to generate the UCM of two offsprings. SBX crossover operator [19] is chosen as the real crossover operator and is applied on the RPM of two parents to obtain the RPM of two offsprings.

\section{Mutation}

1) Swap-window operator: The binary and real versions of this operator are applied separately on UCM and RPM of a chromosome respectively. It works by randomly selecting: a) two units $u_{1}$ and $u_{2}$, b) a time window of width $w$ (hours) between 1 and $T_{\max }$ and c) a window position. The entries of the two units $u_{1}$ and $u_{2}$ included in the window are then exchanged. This operator acts like a sophisticated mutation operator [3].

2) Window mutation operator: This operator works on the UCM of a chromosome by randomly selecting a) a unit, b) a time window of width $w$ (hours) between 1 and $T_{\max }$ and c) a window position. Then it mutates all the bits included in the window, turning all of them to either 1 's or all of them to 0 's with an equal probability [3].

The steps of MOUCA are described as follows:

1. Parameter-setting input: Population size, generation number, Lmax, crossover probability, distribution index for SBX crossover, swap-window operator probability, window mutation operator probability, and penalty values $P_{1}, P_{2}$ and $P_{3}$.

2. Initialization: In the initial population, UCM and RPM of all chromosomes are randomly generated.

3. Repair process and constraint violation evaluation: Heuristic based repair operator is used to repair the chromosomes for load demand equality constraint violation. Constraint violation (min up-down time, load demand and LOLP) is calculated and summed up for each chromosome.

4. Objective function evaluation: UCM and RPM of each chromosome are multiplied to form the resultant power matrix (Res.PM). The objective function $F_{l}$ is then calculated using the Res.PM and objective function $F_{2}$ is equal to the EUE index.

5. Multi-objective ranking: The population is ranked using the constrained-domination principle.

6. Selection: Constrained-binary tournament selection is used to form the mating pool.

7. Crossover: Crossover works by randomly selecting two parent chromosomes from the mating pool. Window crossover operator is applied on the UCM and SBX crossover operator on the RPM of the two parent chromosomes to form the UCM and RPM of the two offspring chromosomes.

8. Mutation: Swap-window operator and windowmutation operator are then applied on the offspring chromosomes.

9. Repair process, constraint violation and objective function evaluation: Steps 3-4 are performed for the offspring population.

10. Multi-objective ranking of combined population: The parent population and offspring population are 
then combined and ranked according to the constrained-domination principle.

11. Replacement: The next generation is formed using the $\mu+\lambda$ elitism principle.

12. Termination: If termination condition is satisfied then obtain trade-off non-dominated solutions else repeat steps (5) to (11).

\section{CASE STUdy AND Discussion}

In this paper, MOUCA is implemented on a test system of 26 thermal generating units [2], considering a dispatch period of 24 hours. The results are compared with the study of Simopoulos et al. [2] where the UC problem was dealt with system operation cost as the single (economic) objective and reliability (LOLP and EUE) as constraint. This case study illustrates the significance of multi-objectivizing the UC problem by setting reliability (EUE) as a helper-objective.

It is noted that Lmax is given in percent (\%) whereas EUE and Emax are expressed as a percentage of the expected energy demand of the total dispatch period. It is noted that Emax is the user defined maximum allowed limit of the EUE reliability index as defined in the benchmark study[2]. The lead time of the system is fixed as 4 hours. All simulations are performed using C platform on a PC with Intel Xeon $2.53 \mathrm{GHz}$ processor and $12 \mathrm{~GB}$ memory. The system and load demand data can be found in [2]. The parameter settings of the algorithm obtained from experiments are as shown in Table I. The computational time requirement of MOUCA is around 40 minutes.

\section{A. Multi-objectivization in the case of uncertainty due to unit unavailability}

The effect of multi-objectivization in UC problem is first investigated by performing simulations considering the uncertainty due to unit unavailability alone. "Pareto-optimal" (P-O) fronts are obtained for no-LOLP constraint, Lmax = $1.5 \%$ and $\operatorname{Lmax}=1.0 \%$ case as shown in Fig. 1. It can be seen from Fig. 1 that as the LOLP constraint gets stricter (i.e., Lmax decreases), the solutions obtained are of lesser EUE objective value and higher cost.

TABLE I

PARAMETER SETTINGS

\begin{tabular}{|c|c|}
\hline Population size & 300 \\
\hline Generation number & 10000 \\
\hline Crossover probability & 0.6 \\
\hline Variable crossover probability in SBX & 1 \\
\hline Distribution index in SBX & 0.2 \\
\hline Swap window operator probability & 0.25 \\
\hline Window mutation operator probability & 0.25 \\
\hline Penalty parameter $P_{I}$ & 100 \\
\hline Penalty parameter $P_{2}$ & 1000 \\
\hline Penalty parameter $P_{3}$ & 100 \\
\hline
\end{tabular}

It is interesting to note from the figure that multiobjectivizing the UC problem by adding reliability as an additional objective, a knee region is explored. This region is important in multi-objective optimization problems. A knee region on the $\mathrm{P}-\mathrm{O}$ front consists of solutions with the maximum marginal rates of return i.e. solutions for which a small improvement in one objective would lead to a large deterioration in the other objective [20]. Such characteristic of the knee solutions make them particularly interesting to decision makers for practical applications [21]. A knee region can be visually identified as a convex bulge in the $\mathrm{P}-\mathrm{O}$ front [20]. According to Das [22], knee on the P-O front corresponds to farthest solution from the line formed by joining the extreme solutions on the P-O front. In Fig. 1, the knee of the P-O front is shown according to the definition given by Das [22]. The neighboring solutions to the knee on the P-O front are called the knee solutions.

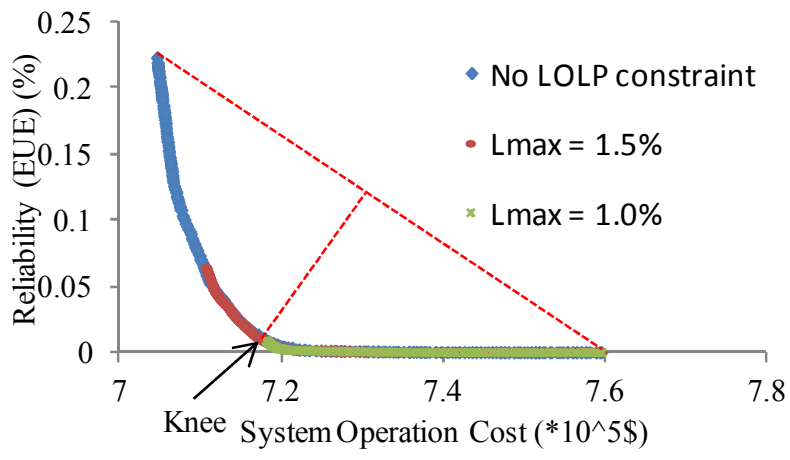

Fig. 1. P-O fronts for system operation cost v/s reliability (EUE) for 26 unit system considering unit unavailability for different Lmax values.

It can be seen from Fig. 1 that a good representation of solutions is obtained on the cost-reliability (EUE) P-O front for no LOLP constraint case. This P-O front offers the system operators to choose any optimum solution from the wider range of cost-reliability trade-off solutions. The corresponding LOLP values for the chosen solution can be determined for every hour. However, when the problem is constrained with $L \max =1.5 \%$ and $1.0 \%$, the region of non-dominated solutions is limited as can be seen in Fig. 1. The Fig. 1 depicts that the knee region lying above the knee is lost if an arbitrary value is assigned for Lmax, as observed with $\operatorname{Lmax}=1.0 \%$.

\section{B. Multi-objectivization in the case of uncertainty due to unit unavailability and load forecast error}

Simulations are further performed by incorporating both unit unavailability and load forecast uncertainties in this study. Fig. 2 shows the P-O fronts corresponding to standard deviation (S) of $1 \%, 3 \%$ and $5 \%$ in load forecast uncertainty and $\operatorname{Lmax}=1.5 \%$. It can be observed that the P-O front obtained for lower $\mathrm{S}$ value dominates the P-O front obtained for higher $\mathrm{S}$ value. The reason behind the shift in $\mathrm{P}-\mathrm{O}$ fronts as the load forecast uncertainty increases can be analyzed from Table II. For example, consider the horizontal line L1 in Fig. 2 that is drawn corresponding to EUE $=0.03$. The solutions just below the line $\mathrm{L} 1$ on the three P-O fronts are compared for cost and spinning reserve. It can be observed from Table II that for a particular EUE objective value (in this example 0.03 ), as the load forecast uncertainty increases; more system spinning reserve gets scheduled automatically to maintain the reliability level (close to $\mathrm{EUE}=0.03$ here) and thus results in higher system operation cost. Similar observations are made from comparisons for EUE $=0.02$ and 0.01 as shown in Table 
II.

To analyze solutions on a particular P-O front, the P-O front corresponding to $\mathrm{S}=1 \%$ is chosen here. Three horizontal lines L1, L2 and L3 are drawn corresponding to EUE $=0.03$, 0.02 and 0.01 . The solutions on the P-O front just below these lines are compared for their cost and spinning reserve. Table II shows that for a particular case of load forecast uncertainty (in this example $\mathrm{S}=1 \%$ ), as the solution's reliability level increases (i.e., EUE value decreases); its scheduled system spinning reserve also increases which results in higher operation cost. Similar observations are also made from comparisons for $\mathrm{S}=3 \%$ and $5 \%$ as shown in Table II.

In the literature, generally most of the studies adopt deterministic criteria for evaluating the system spinning reserve requirements. For example, the most common criteria adopted in practice are that the reserve should be at least equal to the largest capacity of the unit or a specific percentage of the hourly system load. Such deterministic approaches have the disadvantage of not being able to consider the uncertainties in the problem. However, it is noted that the system spinning reserve gets scheduled automatically in the presented approach according to the desired level of reliability as discussed above.

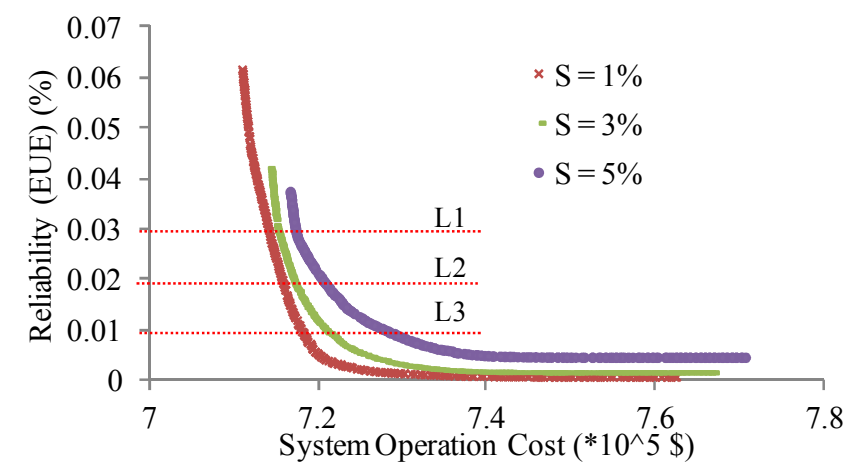

Fig. 2. P-O fronts for system operation cost v/s reliability (EUE) for 26 unit system considering unit unavailability and different values of load forecast uncertainty and $\operatorname{Lmax}=1.5 \%$.

\section{Benchmarking and Discussion}

In this section, the results obtained above are benchmarked against the results presented in the study of Simopoulos et al. [2]. In study [2], the UC problem was solved considering system operation cost as the single objective with reliability (LOLP and EUE) as constraint. The approach used by the authors in study [2] is described in brief as follows. Simulated annealing was used for finding the optimum schedule of the generating units while the economic dispatch problem was solved separately for each hour through a quadratic programming routine.

The significance of multi-objectivizing the UC problem by adding reliability (EUE) as an objective (rather than a constraint) is explained in the following comparison and discussion. For comparison, the P-O front corresponding to $\mathrm{S}$ $=5 \%, \operatorname{Lmax}=1.5 \%$ is chosen here and shown in Fig. 3 . The same problem was solved in study [2] with Emax $=0.05 \%$ constraint. It can be seen that all the solutions contained in the P-O front in Fig. 3 satisfy the constraint of Emax $=0.05 \%$ (marked by a horizontal dotted red line). The region circled in the P-O front is expanded and shown in Fig. 4. In Fig. 4, the vertical line $\mathrm{L} 1$ on the left coincides with the best cost solution (\$716546) obtained from our proposed approach while the vertical line L2 on the right coincides with the best cost solution (\$716862) obtained in the study [2]. It is noted that our result is better by $\$ 316$. Moreover, it is also observed from Fig. 4 that there are many solutions in between the two vertical lines obtained from our proposed multi-objectivized approach which are better than the best cost solution obtained in the study [2].

Additional advantage of setting reliability (EUE) as a helper-objective is that the complete trade-off P-O solutions between system operation cost and reliability (EUE) are obtained in one single run of MOUCA. The P-O front exactly shows the nature of the trade-off between the system operation cost and the reliability (EUE index) and provides the information of the knee region which can be of high interest to the system operator as explained earlier.

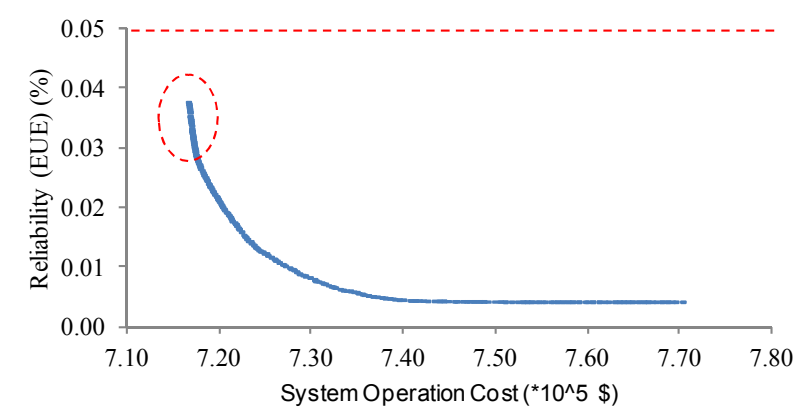

Fig. 3. P-O front for system operation cost v/s reliability (EUE) for 26 unit system considering unit unavailability and $\mathrm{S}=5 \%$ load forecast uncertainty and $\operatorname{Lmax}=1.5 \%$.

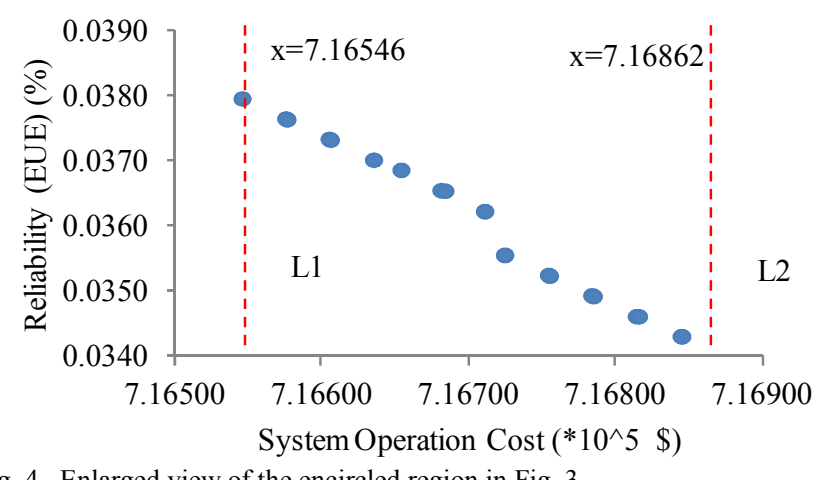

Fig. 4. Enlarged view of the encircled region in Fig. 3

The best cost solution obtained using MOUCA for system operation cost and reliability (EUE) as objectives is compared against the best cost solution obtained for system operation cost as the single objective and reliability (LOLP and EUE) as constraint in study [2]. Ten simulations are run for different cases as shown in Table III and IV. The comparison of results for various reliability levels, considering uncertainty to unit unavailability are shown in Table III. Table IV shows the comparison of results by including both unit unavailability and load forecast uncertainties. It can be observed from Table III and Table IV that MOUCA performed better than the 
benchmark in terms of best cost and average cost in all the cases. The worst cost for the ten runs was also better than the benchmark except in one case. This proves the robustness and the consistency of MOUCA in solving the multi-objectivized UC problem in uncertain environment.

\section{Quantitative Performance Assessment}

In this paper, the quantitative performance assessment of MOUCA has been done using hypervolume indicator [23]. MOUCA is executed for 10 different runs on the system of 26 thermal units for the various cases shown in Fig. 2. Hypervolume indicator signifies the proximity and the spread of non-dominated front with respect to the reference set. The reference set of non-dominated solutions is obtained by choosing the best non-dominated solutions over 10 different runs. The statistical value of this indicator lies between 0 and 1 , where 0 represents the best performance and 1 represents the worst performance. It can be observed in Table $\mathrm{V}$ that the mean value of the hypervolume indicator is close to zero in all the cases which shows a good performance of MOUCA. Moreover, the difference between the best and the worst values as well as the standard deviation of hypervolume indicator is small. This signifies consistent performance of MOUCA over 10 different runs for various cases and proves the efficiency of MOUCA in solving the multi-objectivized $\mathrm{UC}$ problem in uncertain environment.

\section{CONCLUSIONS}

The short-term UC problem is generally solved as a singleobjective optimization problem. In this paper, we presented multi-objectivization of UC problem in uncertain environment by considering reliability as a helper objective and solved the re-formulated multi-objectivized UC problem using our earlier proposed MOEA [1].

The effect of multi-objectivization in UC problem by adding reliability as a helper objective was studied on a test system of 26 thermal generating units. The results obtained using MOUCA were benchmarked against the results presented in study [2] where the UC problem was solved as a reliability-constrained single-objective optimization problem using SA. The comparison demonstrated that MOUCA clearly outperformed the benchmark and could provide not only one but many better solutions (in terms of best cost) than the benchmark. The main reasons behind the success of the proposed multi-objectivized UC approach against the approach presented in study [2] are: 1) the use of populationbased algorithm and 2) the use of helper-objective that being conflicting in nature with the primary objective helps maintain population diversity and guides the search away from local optima. Additional advantage of setting reliability as a helperobjective was that a wide set of trade-off cost-reliability P-O solutions is obtained in one single run of MOUCA. Moreover, the obtained $\mathrm{P}-\mathrm{O}$ front provided the information of the existing knee region for the problem which could be of high interest to the system operators as discussed. The capability of MOUCA in consistently and efficiently solving the UC problem in uncertain environment was displayed not only by comparison against the benchmark but also by performing quantitative performance assessment using hypervolume indicator.
In future, the effect of multi-objectivization of UC problem via helper-objective may be studied by considering other important parameters like emission cost and security as helper-objectives.

\section{ACKNOWLEDGEMENT}

This work is supported by National Research Foundation, NRF-2007EWT-CERP01-0954 (R-263-000-522-272).

\section{REFERENCES}

[1] A. Trivedi, N. M. Pindoriya, D. Srinivasan and D. Sharma, "Improved multi-objective evolutionary algorithm for day-ahead thermal generation scheduling," presented at the IEEE Congress on Evolutionary Computation, New Orleans, USA, 2011.

[2] D. N. Simopoulos, S. D. Kavatza, C. D. Vournas, "Reliability constrained unit commitment using simulated annealing," IEEE Transactions on Power Systems, vol. 21, pp. 1699-706, 2006.

[3] S. A. Kazarlis, A. G. Bakirtzis and V. Petridis, "A genetic algorithm solution to the unit commitment problem," IEEE Transactions on Power Systems, vol. 11, pp. 83-92, 1996.

[4] I. G. Damousis, A. G. Bakirtzis and P. S. Dokopoulos, "A solution to the unit-commitment problem using integer-coded genetic algorithm," IEEE Transactions on Power Systems, vol. 19, pp. $1165-72,2004$.

[5] J. Valenzuela and A. E. Smith, "A seeded memetic algorithm for large unit commitment problems," Journal of Heuristics, vol. 8, pp. 173-95, 2002.

[6] T. O. Ting, M. V. C. Rao and C. K. Loo, "A novel approach for unit commitment problem via an effective hybrid particle swarm optimization," IEEE Transactions on Power Systems, vol. 21, pp. 411-18, 2006.

[7] X. Yuan, A. Su, H. Nie, Y. Yuan and L. Wang, "Unit commitment problem using enhanced particle swarm optimization algorithm," Soft Computing, vol. 15, pp. 139-48, 2011.

[8] A. H. Mantawy, Y. L. Abdel-Magid and S. Z. Selim, "A simulated annealing algorithm for unit commitment," IEEE Transactions on Power Systems, vol. 13, pp. 197-204, 1998.

[9] D. F. Lochtefeld and F. W. Ciarallo, "Multiobjectivization via Helper-Objectives with the Tunable Objectives Problem," 2011.

[10] J. D. Knowles, R. A. Watson and D. Corne, "Reducing local optima in single-objective problems by multi-objectivization," in Evolutionary Multi-Criterion Optimization. First International Conference, EMO 2001, 7-9 March 2001, Berlin, Germany, 2001, pp. 269-83.

[11] M. T. Jensen, "Helper-objectives: Using multi-objective evolutionary algorithms for single-objective optimization," Journal of Mathematical Modelling and Algorithms, vol. 3, pp. 323-347, 2004.

[12] M. Jahne, X. Li and J. Branke, "Evolutionary algorithms and multi-objectivization for the travelling salesman problem," in 11th Annual Genetic and Evolutionary Computation Conference, GECCO-2009, July 8, 2009 - July 12, 2009, Montreal, QC, Canada, 2009, pp. 595-602.

[13] J. Handl and J. Knowles, "An evolutionary approach to multiobjective clustering," IEEE Transactions on Evolutionary Computation, vol. 11, pp. 56-76, 2007.

[14] C. A. C. Coello, "Treating constraints as objectives for singleobjective evolutionary optimization," Engineering Optimization, vol. 32 , pp. 275-308, 2000.

[15] S. Watanabe and K. Sakakibara, "Multi-objective approaches in a single-objective optimization environment," in 2005 IEEE Congress on Evolutionary Computation, IEEE CEC 2005, September 2, 2005 - September 5, 2005, Edinburgh, Scotland, United kingdom, 2005, pp. 1714-1721.

[16] R. N. Allan, R. Billinton and N. M. K. Abdel-Gawad, "The IEEE Reliability Test System-extensions to and evaluation of the generating system," IEEE Transactions on Power Systems, vol. PWRS-1, pp. 1-7, 1986. 

technique for unit commitment problem by extended priority list," IEEE Transactions on Power Systems, vol. 18, pp. 882-888, 2003.

[18] K. Deb, A. Pratap, S. Agarwal and T. Meyarivan, "A fast and elitist multiobjective genetic algorithm: NSGA-II," IEEE Transactions on Evolutionary Computation, vol. 6, pp. 182-97, 2002.

[19] K. Deb, Multi-objective Optimization using Evolutionary Algorithms. Singapore: John Wiley \& Sons, 2001.

[20] L. Rachmawati and D. Srinivasan, "Multiobjective evolutionary algorithm with controllable focus on the knees of the Pareto front," IEEE Transactions on Evolutionary Computation, vol. 13, pp. 81024, 2009.

[21] S. Bechikh, L. B. Said and K. Ghedira, "Searching for knee regions in multi-objective optimization using mobile reference points," in 25th Annual ACM Symposium on Applied Computing, SAC 2010, March 22, 2010 - March 26, 2010, Sierre, Switzerland, 2010, pp. 1118-1125.

[22] I. Das, "On characterizing the 'knee' of the Pareto curve based on normal-boundary intersection," Structural Optimization, vol. 18, pp. 107-115, 1999

[23] E. Zitzler, L. Thiele, M. Laumanns, C. M. Fonesca and V. G. Da Fonesca, "Performance assessment of multiobjective optimizers: An analysis and review," IEEE Transactions on Evolutionary Computation, vol. 7, pp. 117-132, 2003.

TABLE II

COMPARISON OF BEST COST AND SPINNING RESERVE FOR DIFFERENT EUE VALUES AND LOAD FORECAST UNCERTAINTIES

\begin{tabular}{|c|c|c|c|c|c|c|c|c|c|}
\hline \multirow{2}{*}{$\begin{array}{c}\text { EUE } \\
(\%)\end{array}$} & Best Cost & $\begin{array}{c}\text { Spinning } \\
\text { Reserve } \\
(\mathrm{MW})\end{array}$ & $\begin{array}{c}\text { Spinning } \\
\text { Reserve } \\
(\%)\end{array}$ & Best Cost & $\begin{array}{c}\text { Spinning } \\
\text { Reserve } \\
(\mathrm{MW})\end{array}$ & $\begin{array}{c}\text { Spinning } \\
\text { Reserve } \\
(\%)\end{array}$ & Best Cost & $\begin{array}{c}\text { Spinning } \\
\text { Reserve } \\
(\mathrm{MW})\end{array}$ & $\begin{array}{c}\text { Spinning } \\
\text { Reserve } \\
(\%)\end{array}$ \\
\hline 0.03 & 7.142063 & 8188 & 14.9 & 7.149284 & 8482 & 15.4 & 7.172830 & 9271 & 16.9 \\
\hline 0.02 & 7.157435 & 8753 & 15.9 & 7.168709 & 9110 & 16.6 & 7.203927 & 10051 & 18.3 \\
\hline 0.01 & 7.180030 & 9467 & 17.2 & 7.207682 & 10231 & 18.6 & 7.278077 & 11754 & 21.4 \\
\hline
\end{tabular}

TABLE IIII

COMPARISON OF BEST COST AND SPINNING RESERVE FOR DIFFERENT EUE VALUES AND LOAD FORECAST UNCERTAINTIES

\begin{tabular}{|c|c|c|c|c|c|c|c|c|c|c|}
\hline $\begin{array}{c}\text { Emax } \\
(\%)\end{array}$ & $\begin{array}{c}\text { Lmax } \\
(\%)\end{array}$ & $\begin{array}{c}\text { Best } \\
\text { Cost }(\$)\end{array}$ & $\begin{array}{c}\text { Best Cost } \\
(\text { benchmark } \\
(\$)[2]\end{array}$ & $\begin{array}{c}\text { Diff. } \\
(\$)\end{array}$ & $\begin{array}{c}\text { Av. } \\
\text { Cost }(\$)\end{array}$ & $\begin{array}{c}\text { Av. Cost } \\
(\text { benchmark }) \\
(\$)[2]\end{array}$ & $\begin{array}{c}\text { Diff. } \\
(\$)\end{array}$ & $\begin{array}{c}\text { Worst } \\
\text { Cost }(\$)\end{array}$ & $\begin{array}{c}\text { Worst Cost } \\
(\text { benchmark }) \\
(\$)[2]\end{array}$ & $\begin{array}{c}\text { Diff. } \\
(\$)\end{array}$ \\
\hline 0.10 & - & 707998 & 708789 & -791 & 708127 & 709052 & -925 & 708309 & 709471 & -1162 \\
\hline 0.05 & - & 711486 & 712243 & -757 & 711621 & 713411 & -1790 & 711744 & 713787 & -2043 \\
\hline 0.01 & - & 718010 & 718747 & -737 & 718413 & 719078 & -665 & 718758 & 719868 & -1110 \\
\hline- & 1.5 & 709580 & 710696 & -1116 & 710582 & 710971 & -389 & 710749 & 711315 & -566 \\
\hline- & 1.0 & 717874 & 717938 & -64 & 717942 & 718039 & -97 & 718032 & 718135 & -103 \\
\hline 0.05 & 1.5 & 711426 & 712067 & -641 & 711572 & 712704 & -1132 & 711596 & 713473 & -1877 \\
\hline 0.01 & 1.0 & 717874 & 718574 & -700 & 717942 & 718714 & -772 & 718032 & 718827 & -795 \\
\hline
\end{tabular}

TABLE IV

COMPARISON FOR VARIOUS RELIABILITY LEVELS AND LOAD FORECAST UNCERTAINTY VALUES

\begin{tabular}{|c|c|c|c|c|c|c|c|c|c|c|c|}
\hline $\begin{array}{c}\text { Emax } \\
(\%)\end{array}$ & $\begin{array}{c}\text { Lmax } \\
(\%)\end{array}$ & $\begin{array}{c}\text { S } \\
(\%)\end{array}$ & $\begin{array}{c}\text { Best Cost } \\
(\$)\end{array}$ & $\begin{array}{c}\text { Best Cost } \\
(\text { benchmark }) \\
(\$)[2]\end{array}$ & $\begin{array}{c}\text { Diff. } \\
(\$)\end{array}$ & $\begin{array}{c}\text { Av. Cost } \\
(\$)\end{array}$ & $\begin{array}{c}\text { Avg. Cost } \\
(\text { benchmark }) \\
(\$)[2]\end{array}$ & $\begin{array}{c}\text { Diff. } \\
(\$)\end{array}$ & $\begin{array}{c}\text { Worst } \\
\text { Cost }(\$)\end{array}$ & $\begin{array}{c}\text { Worst Cost } \\
(\text { benchmark }) \\
(\$)[2]\end{array}$ & $\begin{array}{c}\text { Diff. } \\
(\$)\end{array}$ \\
\hline 0.05 & 1.5 & 0 & 711426 & 712067 & -641 & 711572 & 712704 & -1132 & 711596 & 713473 & -1877 \\
\hline 0.05 & 1.5 & 1 & 711677 & 712216 & -539 & 711816 & 712384 & -568 & 712903 & 712638 & +265 \\
\hline 0.05 & 1.5 & 3 & 713732 & 713855 & -123 & 713930 & 714119 & -189 & 714258 & 714390 & -132 \\
\hline 0.05 & 1.5 & 5 & 716546 & 716862 & -316 & 716641 & 717098 & -457 & 716677 & 717318 & -641 \\
\hline 0.01 & 1.0 & 0 & 717874 & 718574 & -700 & 717942 & 718714 & -772 & 718032 & 718827 & -795 \\
\hline 0.01 & 1.0 & 1 & 717778 & 718843 & -1065 & 717810 & 719071 & -1261 & 717837 & 719334 & -1497 \\
\hline 0.01 & 1.0 & 3 & 720440 & 722544 & -2104 & 720522 & 722859 & -2337 & 720558 & 723107 & -2549 \\
\hline 0.01 & 1.0 & 5 & 727390 & 730675 & -3285 & 727674 & 731027 & -3353 & 727951 & 731448 & -3497 \\
\hline
\end{tabular}

TABLE V

HYPERVOLUME INDICATOR VALUES

\begin{tabular}{|c|c|c|c|c|c|}
\hline Case & Mean & Median & Best & Worst & Std.Dev \\
\hline $\mathrm{S}=1 \%$ & 0.0027 & 0.0015 & 0.0012 & 0.0139 & 0.0039 \\
\hline $\mathrm{S}=3 \%$ & 0.0023 & 0.0018 & 0.0016 & 0.0036 & 0.0008 \\
\hline $\mathrm{S}=5 \%$ & 0.002 & 0.0019 & 0.0017 & 0.0023 & 0.0002 \\
\hline
\end{tabular}

